(Listed geographically from east to west)

1. School of Education, University of Waikato, Hamilton, New Zealand. H 1

2. Faculty of Education, Monash University, Clayton, Victoria, 3168, Australia.

F 1

3. Centre for Advancement of Teaching, Macquarie University, North Ryde, New South Wales 2113, Australia.

H 2, R 1

4. Faculty of Education, University of Western Australia, Nedlands, Western Australia 6009, Australia.

A 1

5. Department of Education, University of Papua and New Guinea, P.O. Box 1144, Boroko, Territory of Papua \& New Guinea.

C $1, F 2, F 3, F$ 4, F 5, F 6, F 7, F 8, F 9, F 10, F 11, F 12, I 1, l 2, L 1, Q 1

6. Department of Education; University of Hong Kong, Pokfulam Road, Hong Kong.

A 2, C 2, E 1, F 13, F 14, F 15, F 16, F 17, F 18, F 19, F 20, G l, $\mathrm{H} 3, \mathrm{I} 3, \mathrm{~J} 1, \mathrm{~K} 1, \mathrm{~K} 2, \mathrm{Q} 2$

7. School of Education, Chinese University of Hong Kong Shatin, New Territories, Hong Kong.

R 2

8. Hong Kong Association for Mentally Handicapped Children \& Young

Persons, 702 Fung House, Connaught Road C., Hong Kong.

N 1

9. School of Education, University of Singapore, Singapore.

B 14

10. Centre for Educational Services, University of Penang, Minden, Penang, Malaysia.

A $3, \mathrm{~K} 3$

11. National Council for Educational Research and Training, Mehrauli Road, New Delhi 16, India.

C 3 
12. Department of Education, Banaras Hindu University, Kamachha, Varanasi, U.P., India.

G 2

13. Central Institute of Education, University of Delhi, 33 Chhatra Marg, New Delhi 7, India.

M 1

14. Centre for Advanced Study in Education/Faculty of Education and Psychology, Maharaja Sayajirao University, Lokmanya Tilak Road, Baroda -2 , India.

A $4, \mathrm{D} 1, \mathrm{~F} 21, \mathrm{I} 4$

15. Department of Postgraduate Studies and Research in Education, University of Mysore, Mysore State, India.

E 2, J 3, J 4

16. South India Teachers' Union, Council of Educational Research, 95-A, Ramakrishna Mutt Road, Madras 28, India.

F 22, I 5, J 2

17. Institute of Education and Research, University of the Punjab, New Campas, Lahore, Pakistan.

A 15, A $16, \mathrm{H} 22$

18. Institute of Education, University of Sind, Hyderabad, Pakistan. B 15 , B 16, C 25

19. University of Nairobi, P.O. Box 30197, Nairobi, Kenya.

(i) Faculty of Education.

A 5, B 1, F 23, H 4, H 5, M 2

(ii) Child Development Research Unit.

$\mathrm{E} 3, \mathrm{E} \mathrm{4,E} \mathrm{5,} \mathrm{E} \mathrm{5,} \mathrm{E} 7$

(iii) Institute of Development Studies.

O 1

20. Makerere University, P.O. Box 7062, Kampala, Uganda.

(i) Audio-Visual Aids Centre/National Institute of Education.

F 24, I 6, I 7, I 8, P 1

(ii) Department of Education .

A 6, B 2, B 5, B 6, E 15, F 25, F 26, F 27, F 28, G 4,

$\mathrm{H} \mathrm{6,} \mathrm{H} \mathrm{7,} \mathrm{H} \mathrm{8,} \mathrm{H} \mathrm{9,} \mathrm{H} \mathrm{10,} \mathrm{H} \mathrm{11,} \mathrm{H} \mathrm{12,} \mathrm{H} \mathrm{13,} \mathrm{I} \mathrm{9,} \mathrm{I} \mathrm{10,} \mathrm{R} 7$

(iii) Department of Educational Psychology.

C 4, C 5, C 6, C 7, C 8, C 9, C 10, E 8, E 9, E 10, E 11,

E 12, E 13, E 14, G 3, J 5, J 6, K 4

(iv) Department of Language Methods.

F 29, F 30, F 3l, F32

(v) Department of Social Work and Social Administration.

R 3 185 
21. Makerere Institute of Social Research, Makerere University, P.O. Box 7062, Kampala, Uganda.

$\mathrm{B} 3, \mathrm{~B} 4, \mathrm{D} 2, \mathrm{M} 3, \mathrm{R} 4, \mathrm{R} 5, \mathrm{R} 6$

22. University of Zambia, P.O. Box 2379, Lusaka, Zambia.

(i) Department of Education .

A 7

(ii) School of Education.

P 2

(iii) Science Education Research Centre, School of Education .

H 14

(iv) Human Development Research Institute.

E 16

(v) Institute for African Studies.

I 11

23. University of Malawi, P.O. Box 496, Limbe, Malawi.

(i) Soche Hill College.

C 11, F 33

(ii) Chancellor College - Department of History.

B 7

24. Ahmadu Bello University, Zaria, Nigeria.

(i) Department of Education .

H 17

(ii) Adult Education and General Extension Services Unit.

$M 4, M 5, M 6$

25. Department and Institute of Education, University of Ibadan, Ibadan, Nigeria.

C $20, \mathrm{C} 21, \mathrm{C} 22, \mathrm{E} 17, \mathrm{E} 18, \mathrm{~J} 8, \mathrm{R} 11$

26. University of Ife, Ile-Ife, Nigeria.

(i) Institute of Education.

G 5 , H 18

(ii) Faculty of Education.

D 3, E 19, H 19, M 7

27. University of Lagos, Lagos, Nigeria.

(i) College of Education .

$\mathrm{B} 8, \mathrm{~F} 34, \mathrm{H} 15, \mathrm{~J} 7, \mathrm{~L} 2, \mathrm{~L} 3, \mathrm{R} 8, \mathrm{R} 9$ 
(ii) Comparative Education Study and Adaptation Centre.

H 16

28. The West African Examinations Council, Test Development and Research Office, Private Mail Bag 1076, Yaba, Lagos, Nigeria.

C 12, C 13, C 14, C 15, C 16, C 17, C 18, C 19, C 23, J 9, J 10, $\mathrm{J} 11, \mathrm{~J} 12, \mathrm{R} 10$

29. The Ghana Association of Science Teachers, Ministry of Education, P.O. Box M.45, Accra, Ghana.

F 35

30. Njala University College, University of Sierra Leone, Private Mail Bag, Freetown, Sierra Leone.

C $23, \mathrm{~J} 13$

(i) Department of Geography and Environmental Studies.

F 36

(ii) Department of Teacher Education.

$\mathrm{H} 20$

31. The Commonwealth Secretariat, Marlborough House, Pall Mall, London, SW1Y SHX, Britain.

D $5, \mathrm{~N} 2$

32. University of London Institute of Education, Malet Street, London, W.C.1, Britain.

(i) Department of Education in Tropical Areas .

A $9, \mathrm{D} 5, \mathrm{~N} 2$

(ii) lnstitute of Commonwealth Studies .

R 12

33. Higher Research Unit, London School of Economics, Houghton Street, Aldwych, London WC2A $2 \mathrm{AE}$, Britain.

\section{A 8}

34. Centre for West African Studies, Birmingham University, P.O. Box 363, Birmingham 15, Britain.

A $10, \mathrm{~K} 5$

35. Project Planning Centre for Developing Countries, Univer'sity of Bradford, Great Horton Road, Bradford 7, Britain.

O 2

36. Department of Psychology, University of Bristol, Bristol 2, Britain. F 37, F 38, F 39 
37. Department of Education, University College Cardiff, P.O. Box 78, Cardiff CF1 1 XL, Britain.

J 14

38. Department of Educational Studies, University of Edinburgh, 11 Buccleuch P'lace, Edinburgh EH8 9JT, Britain.

$\mathrm{M} \mathrm{8,O} \mathrm{3,} \mathrm{R} 14$

39. Department of Education, University of Exeter, Exeter, EX4 4QJ, Britain.

R 13

40. Department of International Economic Studies, University of Glasgow, Glasgow W.2, Britain.

K 7

41. Department of Educational Studies, University of Hull, 173 Cottingham Road, Hull, Britain.

A 11

42. Institute of Education, University of Keele, Keele, Staffordshire, ST5 5BG, Britain.

[ 12

43. Department of Government, University of Manchester, Oxford Road, Manchester 13, Britain.

Q.3

44. University of Oxford, Oxford, Britain.

(1) Queen Elizabeth House.

$\wedge 12, \mathrm{~K} 6$

(ii) Institute of Economics and Statistics.

$\Lambda 13$

45. School of Education, University of Reading, Whiteknights, Reading, Berkshire, Britain.

B 9, I 13

46. School of Education, The University, Southampton, SO9 5NH, Britain.

I 14

47. Department of Education, University College of Swansea, Singleton Park, Swansea, Glamorgan, SA2 8PP, Britain.

I. 4

48. Department of Language, University of York, York, Britain.

II 23 
49. Department of Education, University of the West Indies, Mona, Kingston 7, Jamaica, W.I..

E $20, \mathrm{G} 8$

50. Institute of Education, University of the West Indies, St. Augustine, Trinidad, W.I. .

G $7, \mathrm{H} 21, \mathrm{~J} 15$

51. Faculty of Education, University of Guyana, Box 841, Georgetown, Guyana, South America.

G 6

52. Department of History, Univer sity of Wisconsin, Madison, Wisconsin, 53706, U.S.A..

B 10

53. University of Calgary, Calgary 44, Alberta, Canada.

(i) Faculty of Education/Department of Educational

Foundations.

A $14, \mathrm{~B} 11, \mathrm{~B} 12, \mathrm{D} 4$

54. McGill University, Montreal 110, P.Q., Canada.

(i) Faculty of Education.

B 13

55. Department of Adult Education, Ontario Institute for Studies in Education, 102 Bloor Street West, Toronto 5, Ontario, Canada. C $24, \mathrm{M} 9, \mathrm{M} 10$ 


\section{AUTHOR INDEX}

Adam, Dr. R . A 1

Ademuwagun, Dr. Z.A. R 8

Battle, V.M.

B 4

Adeosun, D.O.O.

03

Beckett, B.S.

F 13

Adeyinka, J.A.

M 7

Bentt, V.M.

G 6

Adu-Ampora, S.M.

F 35

Bevli, U.K.

C 3

Afolayan, Dr. A.

H 18

Bhagia, S.

A 4

Afzal, M.

H 22

Bibby, J.

K 5

Agard, J.

F 25

Blaug, $M$.

A 8

Agboro, A.A.

C 17

Bolam, D.W.

l 12

Ahmad, B.

A 8

Bortei-Doku, S.

F 35

Ahmad, $S$.

B 16

Bown, Prof. L.

M 4

Ahmad, Dr. Z.

A 15, A 16

Brooksbank, $M$.

112

Ahluwalia, Dr. S.P.

G 2

Buch, Prof. M.B.

A $4, F 21$

Akeju, Dr. S.S.A.

C $12, \mathrm{C} 13$,

C 14, C 23, J $11, \mathrm{~J} 12$,

Buch, P.M.

A 4

D 3, H 19

Akinpelu, Dr. J.A.

C 19

Akpabio, U.H.

$\mathrm{H} 4$

Andahwa, E.S.

$\mathrm{H}$
$\mathrm{K} 7$

Callaway, Prof. A.

A $12, \mathrm{~K} 6$

Caygill, $M$.

R 12

Anderson, J.

K 7

Chandrasekharaiah, Dr. K.V.B 13

Anderson, J.W.

E 8

Anderson, R.

F 37

Ansari, Dr. N.A.

M 1

Chang, P.M.P.

A 3

Arunajatai, V.

J 2

Arye, J.R.

R 10

Chatterji, Nr.B.B.

C 3

Asinge, D.

A. 11

Clarke, R.F.

C 8

Asiedu, R.

I 12

Awang, A.B.

K 3

Craig, D.R.

E $20, G 8$

Chick, J.D.

Q 3

Awuku, K.A.

F 35

Dart, Prof.F.E.

F 2

Datta, Dr. A.K.

P 2

Desai, Prof. D.M.

A 4

De Benko, Dr. E.

R 4

Denys, L.O.

C $24, \mathrm{M} 9$

Donohue, $\mathrm{M}$.

F 9, I 2

Baird, C.L.

G 6

Draper, Dr. J.A.

M. 10

Bakare, Dr.C.G.M.

C $20, C 21$,

D'Souza, W.

F 29

C 22, E 17 , Durojaiye, M.O.A.

Baloch, Dr. N.A.

B 15

Barki, B.G.

J 4

Barry, D.

F 26

Durojaiye, S.M.

E $9, \mathrm{E} 10$

E $11, \mathrm{G} 3$

K 4

Bates, D.

F 35

Duraiswamy, $M$.

F $30, F 31$

F 22, I 5 


\begin{tabular}{|c|c|c|c|}
\hline $\begin{array}{l}\text { Eedle, J.H. } \\
\text { El-Abd, Prof, H. }\end{array}$ & $\begin{array}{l}\text { D } 5, \mathrm{~N} 2 \\
\mathrm{C} 4, \mathrm{C} 5, \\
\mathrm{C} 6, \mathrm{C} 7, \\
\mathrm{E} 12, \mathrm{E} 13, \\
\mathrm{~J} 5\end{array}$ & $\begin{array}{l}\text { Heyman, Dr. R.D. } \\
\text { Hopcraft, P.N. } \\
\text { Hoorweg, J.C. } \\
\text { Hu, Prof, C.T. }\end{array}$ & $\begin{array}{llll}\mathrm{B} & 11, \mathrm{~B} & 12 \\
\mathrm{O} & 1 & & \\
\mathrm{R} & 6 & & \\
\mathrm{R} & 2 & & \end{array}$ \\
\hline Elliot, Dr. J. & A 7 & & \\
\hline Elston, Rev. P. & B 7 & I lyas, Dr. M. & $\mathrm{H} 22$ \\
\hline English, P.G. & E 15 & $\begin{array}{l}\text { l soardi, D. } \\
\text { Iyengar, R.S. }\end{array}$ & $\begin{array}{l}\text { F } 3,11 \\
\text { I } 5\end{array}$ \\
\hline Fafunwa, Prof. A.B. & $\mathrm{H} 18, \mathrm{M} 7$ & & \\
\hline Falade, G. & I 12 & Jenkins, D.R. & I 12 \\
\hline Fagbongbe, E.O. & L 2 & Johns, G. & J 14 \\
\hline Fiks, Dr. A.I. & $\begin{array}{l}\text { C } 15, \text { C } 16, \\
\text { C } 17, \text { C } 18, \\
\text { C } 19, \text { J } 9, \\
\text { J } 10, \text { R } 10\end{array}$ & $\begin{array}{l}\text { Johnson, R.K. } \\
\text { Johnson, T.J. } \\
\text { Jones, M.D. }\end{array}$ & $\begin{array}{ll}\mathrm{F} & 4 \\
\mathrm{R} & 12 \\
\mathrm{R} & 5\end{array}$ \\
\hline Fisher-Short, W. & I 3 & & \\
\hline Lorrest, R. & I 1 & Kagia, R. & E 4 \\
\hline F reeman, J.M. & F 27 & Kajubi, S.N. & E 14 \\
\hline Fung, H.M. & $\mathrm{R} 2$ & Kale, Dr. S.V. & C 3 \\
\hline Fung, Y.W. & J 1 & $\mathrm{Kan}, \mathrm{Z}$ & I 12 \\
\hline Furlonge, Dr. E.A. & $\mathrm{G} 7, \mathrm{H} 21, \mathrm{~J} 15$ & $\begin{array}{l}\text { Kannade, H.M. } \\
\text { Kapadia, G.G. }\end{array}$ & $\begin{array}{l}\text { C } 3 \\
\text { I } 4\end{array}$ \\
\hline Gardner, P.L. & F 1 & Kent, G. & 114 \\
\hline Gaitirira, L. & E 4 & Ker, A.D.R. & H 9 \\
\hline George, Dr. E.I. & C 3 & Kimilu, D.N. & $\mathrm{F} 23$ \\
\hline Getena, J. & E 3, E 4 & Knight, J.B. & A 13 \\
\hline Ghosh, E.S.K. & F 38 & Krishnamurthy, V. & I 4 \\
\hline Gornes, A. & H 5 & Krishnamurthy, P.V. & I 5 \\
\hline Gunn, A.L. & $\mathrm{H} 8, \mathrm{I} 9$ & Kumaraswamy, T.S. & E 2 \\
\hline Gwayambadde, Y.M.K. & $\mathrm{R} 3$ & & \\
\hline Gwee, Y.H. & B 14 & Lam, L. & $\mathrm{R} 2$ \\
\hline & & Law, P.M. & $\mathrm{R} 2$ \\
\hline Harbottle, M. & H 6 & Lawless, C.J. & F 33, I 13 \\
\hline Harrold, M.S. & F 39 & Lawson, Dr. R.F. & B 12 \\
\hline Hartley, G. & I 12 & Lieber, J.W. & $\mathrm{R} 11$ \\
\hline Hawkes, C.N. & H 23 & Lockwocd, F.G. & $C^{\prime} 11$ \\
\hline Hayward, P. & F 11 & Logan, R.D. & E.3, E 4, \\
\hline Henderson, Dr. N.K. & $\begin{array}{l}\mathrm{C} 2, \mathrm{E} 1, \mathrm{I} 23, \\
\mathrm{~J} 1, \mathrm{~K} 1, \mathrm{~K} 2 \\
\mathrm{Q} 2\end{array}$ & Lohia, R. & $\begin{array}{l}\text { E } 5 \\
\text { F } 10\end{array}$ \\
\hline
\end{tabular}




\begin{tabular}{|c|c|c|c|}
\hline \multirow{3}{*}{$\begin{array}{l}\text { Lucas, Prof. E. } \\
\text { Lulla, T.P. }\end{array}$} & B 2 & Omer, Dr. Z.Z. & H 22 \\
\hline & F 21 & Onwuka, U. & A 9 \\
\hline & & Osiyale, A. & H 15 \\
\hline Maas, J.V.L. & B 3 & Osibodu, B.O. & E 19 \\
\hline Macpherson, T.J.A. & H 7 & Otaala, B. & C 9 \\
\hline Macauley, J.I. & H 18 & Cxlade, D. & B 1 \\
\hline Malani, I. & C 3 & Oyerinde, T.O. & C 18 \\
\hline Malik, Dr. D.M. & H 22 & Ozoro, 0 & H 16 \\
\hline Mazinga, T.M. & F $28, \mathrm{H} 13$ & & \\
\hline Mc Dowell, I. & $\mathrm{R} 6$ & Parameswaran, Dr. E.G. & C 3 \\
\hline McInerney, Prof. M. & G 6 & Parker, S.J. & I 12 \\
\hline Mehta, Dr. P.H. & C 3 & Pearson, Dr. R.E. & F 35 \\
\hline Meyer, Dr. G.R. & $\mathrm{H} 2, \mathrm{R} 1$ & Peil, Dr. M. & A $10, \mathrm{~K} 5$ \\
\hline Milner, S.R. & $\mathrm{F} 5, \mathrm{~F} 10$ & Powell, Dr. B. & G 7,H 21, \\
\hline Mirza, M.S. & A 16 & & \\
\hline Misbahuddin. & C. 25 & Powell, Dr. J.P. & Q 1 \\
\hline Niunroe, R. & E 6 & Pratt, C.A. & $\begin{array}{l}\mathrm{F} 24, \mathrm{I} 6, \\
\mathrm{I} 7, \mathrm{I} 8, \mathrm{P}\end{array}$ \\
\hline Muizzuddin, Dr. S. & H 22 & & \\
\hline Mytton, G. & I 11 & Qurashi, Z.M. & F 21 \\
\hline Natarajan, S. & F 22, I 5 & Radcliffe, Prof. D.J. & B 10 \\
\hline $\mathrm{Ng}$, Dr. J.L. & $\mathrm{R} 2$ & Rai, K. & A 4 \\
\hline Nguru, G.M. & A 5 & Rao, Dr. R.S. & $\mathrm{J} 2$ \\
\hline Nickelson, Dr. A.L. & H 16 & Rashid, Dr. & C 25 \\
\hline Nsereko-Gyagenda, $\mathrm{T}$. & J 6 & Reynolds, P.D. & $\mathrm{F} \quad 14, \mathrm{~F} \quad 15$ \\
\hline Nsibambi, R. & F 32 & Ridgeway, $\mathrm{N}$. & $\mathrm{F} 10, \mathrm{I} 1$ \\
\hline Obe, Dr. E.O. & J 7 & Ritchie, Prof. J.E. & $\begin{array}{llll}H & i \\
F & 10 & F & 11\end{array}$ \\
\hline Obioha, N.E. & $\mathrm{H} 14$ & Russell, D.D. & M 8 \\
\hline Ocitti, J.P. & $\begin{array}{ll}\mathrm{H} & 10, \mathrm{H} 11, \\
\mathrm{H} & 12\end{array}$ & & \\
\hline Ohuche, Dr. R.O. & C 23, H 20 , & Sachadeva, C. & c 3 \\
\hline & J 13 & Salama, Dr. G. & $M 5, M 6$ \\
\hline Ohuche, N. & J 13 & Sanders, A.C.E. & C 10 \\
\hline Okach, J.N. & M 2 & Santhanam, M.R. & F 21 \\
\hline Okonji, O.M. & E 16 & Seeley, S. & E 6 \\
\hline Okunrotifa, P.O. & H 17 & Seetharamu, A.S. & J 3 \\
\hline Olayinka, Dr. M.S. & L 3 & Shah, A. & C 25 \\
\hline Oloyede, A.O. & J 10 & Shah, Dr. G.B. & $\mathrm{D} 1, \mathrm{I} 4$ \\
\hline
\end{tabular}




\begin{tabular}{|c|c|c|c|}
\hline Sharma, M.L. & D 1 & Williams, A.R. & L 4 \\
\hline Simpson, Dr. R.F. & $\begin{array}{l}\text { A } 2, F 16, \\
\text { G } 1\end{array}$ & Williams, Dr. I.W. & $\underset{\mathrm{J}}{\mathrm{G}} 75, \mathrm{H} 21$, \\
\hline Singh, Dr. A.K. & C 3 & Wilson, A. & F 12 \\
\hline Singh, Prof. R.P. & $\mathrm{E} 2, \mathrm{~J} 3, \mathrm{~J} .4$ & Wilson, F.A. & 02 \\
\hline Smail, J.R.W. & B 10 & Wilson, J.M. & $\mathrm{F} \quad 11, \mathrm{~F} 12$ \\
\hline Sokoya, J.A.F. & G $5, H \quad 18$ & Wingfield, R. & I 1 \\
\hline Somade, Chief, B. & $\mathrm{H} 16$ & Withall, Prof. J. & H 16 \\
\hline Srinivasachari, G. & F 22, I 5 & Whiting, B.B. & E $3, E .7$ \\
\hline Ssekamwa, J.C. & B 5 & & \\
\hline Sweeting, A.E. & F 17 & Yapp, H. & F $19, F .20$ \\
\hline Syed, Prof. A.H. & $\begin{array}{l}\text { A } 15, \text { A } 16 \text {, } \\
\text { H } 22\end{array}$ & Yeung, $M$. & N. 1 \\
\hline Taiwo, A.A. & M 7 & Zachariah, Dr. M. & $\begin{array}{ll}\text { A } & 14, \text { B } 12, \\
\text { D } 4\end{array}$ \\
\hline Taiwo, Prof. C.O. & $\begin{array}{l}\text { B 8,F 34, } \\
\text { R 9 }\end{array}$ & & \\
\hline Tam, A. & F $18, \mathrm{H} 3$ & & \\
\hline Tang, F.C. & $\mathrm{N} 1$ & & \\
\hline Tawia, M. & F 35 & & \\
\hline Taylor, S.F. & F 36 & & \\
\hline Thompson, $\mathrm{H}$. & $\begin{array}{l}\mathrm{G} 7, \mathrm{H} 21, \\
\mathrm{~J} 15\end{array}$ & & \\
\hline Tirtha, Dr. N.V. & C 3 & & \\
\hline Townsend, A. & I 12 & & \\
\hline Trevaskis, Dr. G.A. & 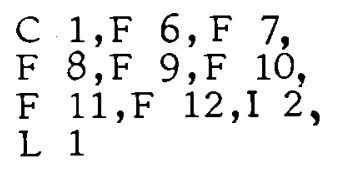 & & \\
\hline Trevor, J. & R 13 & & \\
\hline Trotman, J. & G 6 & & \\
\hline Wallace, T. & M 3 & & \\
\hline Wamai, M. & E. 3 & & \\
\hline Wandira, Prof. A. & A $6, G \cdot 4, R 7$ & & \\
\hline Wass, P.G.L. & $\mathrm{R} 14$ & & \\
\hline Watson, J.K.P. & B 9 & & \\
\hline Watson, $\mathrm{T}$ & B 6, I 10 & & \\
\hline Weeks, Dr. S.G. & $\mathrm{D} 2, \mathrm{M} 3$ & & \\
\hline Whelan, I. & H 1 & & \\
\hline
\end{tabular}

